\title{
Determination of the Load Acting on the Axial Bearing of a Slewing Platform Drive in Hydraulic Excavators
}

\author{
Vesna D. Jovanović ${ }^{1}$, Dragoslav B. Janošević ${ }^{1}$, \\ Dragan Z. Marinković ${ }^{2}$ \\ ${ }^{1}$ University of Niš, Faculty of Mechanical Engineering, A. Medvedeva 14, \\ 18000 Niš, Serbia, vesna.nikolic@ masfak.ni.ac.rs, janos@masfak.ni.ac.rs \\ ${ }^{2}$ Technical University Berlin, Institute of Mechanics, 17. Juni 135, \\ 10623 Berlin, Germany, Dragan.Marinkovic@TU-Berlin.de
}

Abstract: The paper presents a general selection procedure for an axial bearing of a slewing platform drive in hydraulic excavators based on the spectrum of equivalent bearing loads. A mathematical model of an excavator, with a backhoe and a shovel attachment, is defined to determine the spectrum of bearing loads on the basis of possible digging resistances specified in the entire working range of the excavator. As an example, by using the developed software, the size of an axial bearing was selected for a slewing platform drive in a hydraulic excavator with the mass of 100,000 kg, according to the spectrums of equivalent bearing loads obtained from the analysis of an excavator with a backhoe and shovel attachment.

Keywords: axial bearing; hydraulic excavators; slewing platform

\section{Introduction}

Hydraulic excavators perform their primary function of digging through a general configuration of the kinematic chain which consists of the support and movement mechanism $L_{1}$, Figure $1 \mathrm{~b}, \mathrm{c}$, slewing platform $L_{2}$, and changeable multi-member manipulators $L_{m}$, which can be equipped with numerous tools in the form of buckets, claws, grapples, tillers, hammers, hooks, shown in Figure 1a. For digging operations below the ground level, the toward oneself technology (in relation to the excavator operator) is employed and a backhoe attachment is used, shown in Figure $1 \mathrm{~b}$. For digging operations above the ground level the away from oneself technology and a shovel attachment are used, shown in Figure 1c. Hydraulic excavators perform spatial manipulation using the slewing platform $L_{2}$, Figure $1 \mathrm{~b}, \mathrm{c}$, which is attached to the support and movement mechanism $L_{l}$ by way of a 
rotary joint, of the fifth class, in the form of an axial bearing. The slewing drive mechanism of the platform consists of a hydraulic motor 1 as shown in Figure 1d, a reducer 2 coupled over an output gear 2.1 with a ring gear of an axial bearing 3.1. By rule, an axial bearing 3 in Figure 1d consists of an inner ring gear 3.1, which is bolted to the support and movement mechanism $L_{l}$, and a toothless outer ring 3.2, which is bolted to the slewing platform $L_{2}$. Rolling elements (balls, rollers) are positioned between the rings in one or more races. The synthesis of the complete drive mechanism of a hydraulic excavator slewing platform is performed by the following procedure: a) selection of the concept drive solution, b) selection of the axial bearing, c) definition of attachment elements and elements of the support structure to which the bearing is attached, d) selection of the hydraulic motor and slewing drive reducer.

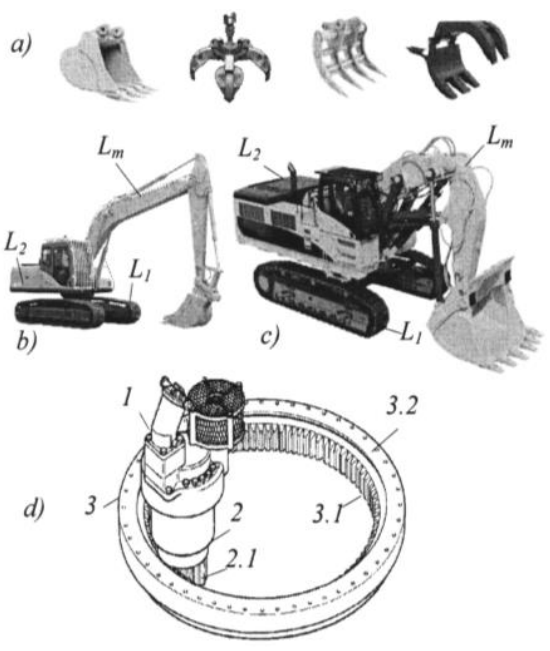

Figure 1

Hydraulic excavators: a) working tools, b) with backhoe attachment

c) with shovel attachment, d) drive mechanism of a hydraulic excavator slewing platform

In the design of the basic excavator systems, research were conducted into: a) analytical modelling and experimental determination of load during the digging process [1] and [2], b) development of mathematical models for kinematic and dynamic excavator analysis [3] and [4], c) development of drive mechanisms and control systems [5] and [6], and d) definition of indicators for analysis and evaluation of excavator digging efficiency [7]. Research into slewing drive mechanisms of excavator platforms deal with: a) loads of axial bearing rolling elements [8] to [10], b) analysis of axial bearing loads in excavators with excavating manipulators [11], and c) regulation of angular velocity of a slewing platform [12] to [14]. This paper provides a selection procedure for the size of an axial bearing of a slewing platform drive in hydraulic excavators with a backhoe and a shovel attachment, based on the spectrum of equivalent bearing loads. 


\section{Mathematical Model of the Excavator}

The mathematical model of the excavator comprises the model of the kinematic chain and the mathematical models of excavator drive mechanisms. The mathematical model encompasses a five-member configuration of the excavator kinematic chain comprising: support and movement mechanism $L_{l}$ in Figure 2, slewing platform $L_{2}$, and a three-member planar attachment with: boom $L_{3}$, stick $L_{4}$ and bucket $L_{5}$. The space of the excavator model is determined with an absolute coordinate system $O X Y Z$ and unit vectors. The excavator support surface lies in the horizontal plane $O X Z$ of the absolute coordinate system, while the vertical axis $O Y$ of the same system overlaps with the axis of the axial bearing of the slewing platform drive mechanism. Members of the excavator kinematic chain compose kinematic pairs of the fifth class - rotary joints with one degree of freedom. The centre of joint $\mathrm{O}_{2}$ of the kinematic pair composed of the support and movement mechanism and the slewing platform is the point of perpendicular intersection of the vertical axis of the joint through the horizontal plane where the centres of rolling elements of the slewing platform drive mechanism axial bearing are positioned. The centres of manipulator joints $O_{i}$ are points of intersection of the horizontal axis of joints through the plans of symmetry of the excavator manipulator kinematic chain. The intersection of the bucket cutting edge through the plane of manipulator represents the centre of the bucket cutting edge $O w$.

The mathematical model of an excavator with a backhoe attachment is defined in the following section.

Each member of the excavator kinematic chain $L_{i}$ is determined, in its local coordinate system $O_{i} x_{i} y_{i} z_{i}$, with a set of quantities:

$L_{i}=\left\{\hat{\vec{e}}_{i}, \widehat{\vec{s}}_{i}, \hat{\vec{t}}_{i}, m_{i}\right\}$

where: $\overrightarrow{\vec{e}}_{i}$ - the unit vector of joint $O_{i}$ axis which connects member $L_{i}$ to the previous member $L_{i-1}, \hat{\vec{s}}_{i}$ - the vector of the position of joint $O_{i+1}$ centre which is used to connect the chain member $L_{i}$ to the next member $L_{i+1}$ (vector si magnitude represents the kinematic length of the member), $\overrightarrow{\vec{t}}_{i}$ - the vector of the position of the member mass centre, $m_{i}$ - the member mass. Quantities marked with a 'cap' above the symbol are determined in the local coordinate system of the member.

The mathematical model of the excavator drive system encompasses the drive mechanisms of manipulator boom, stick, and bucket, which have two-way hydraulic cylinders $c_{3}, c_{4}$, and $c_{5}$ as actuators in Figure 2. Each drive mechanism $C_{i}$ of the excavator manipulator is determined using a set of quantities:

$C_{i}=\left\{d_{i 1}, d_{i 2}, c_{i p}, c_{i k}, \widehat{\vec{a}}_{i}, \widehat{\vec{b}}_{i}, m_{c i}, n_{c i}\right\} \quad \forall i=3,4,5$ 
where: $d_{i 1}, d_{i 2}$ - the diameter of the piston and piston rod of the hydraulic cylinder, $c_{i p}, c_{i k}$ - the initial and final length of the hydraulic cylinder, where: $d_{i l}, d_{i 2}$ - the diameter of the piston and piston rod of the hydraulic cylinder, $c_{i p}, c_{i k}$ - the initial and final length of the hydraulic cylinder, $\overrightarrow{\vec{a}}_{i}, \overrightarrow{\vec{b}}_{i}$ - the vectors of the position of joint centres where the hydraulic cylinder is connected to the kinematic chain members, $m_{c i}$ - the mass of the hydraulic cylinder, $n_{c i}$ - the number of hydraulic cylinders of the drive mechanism.
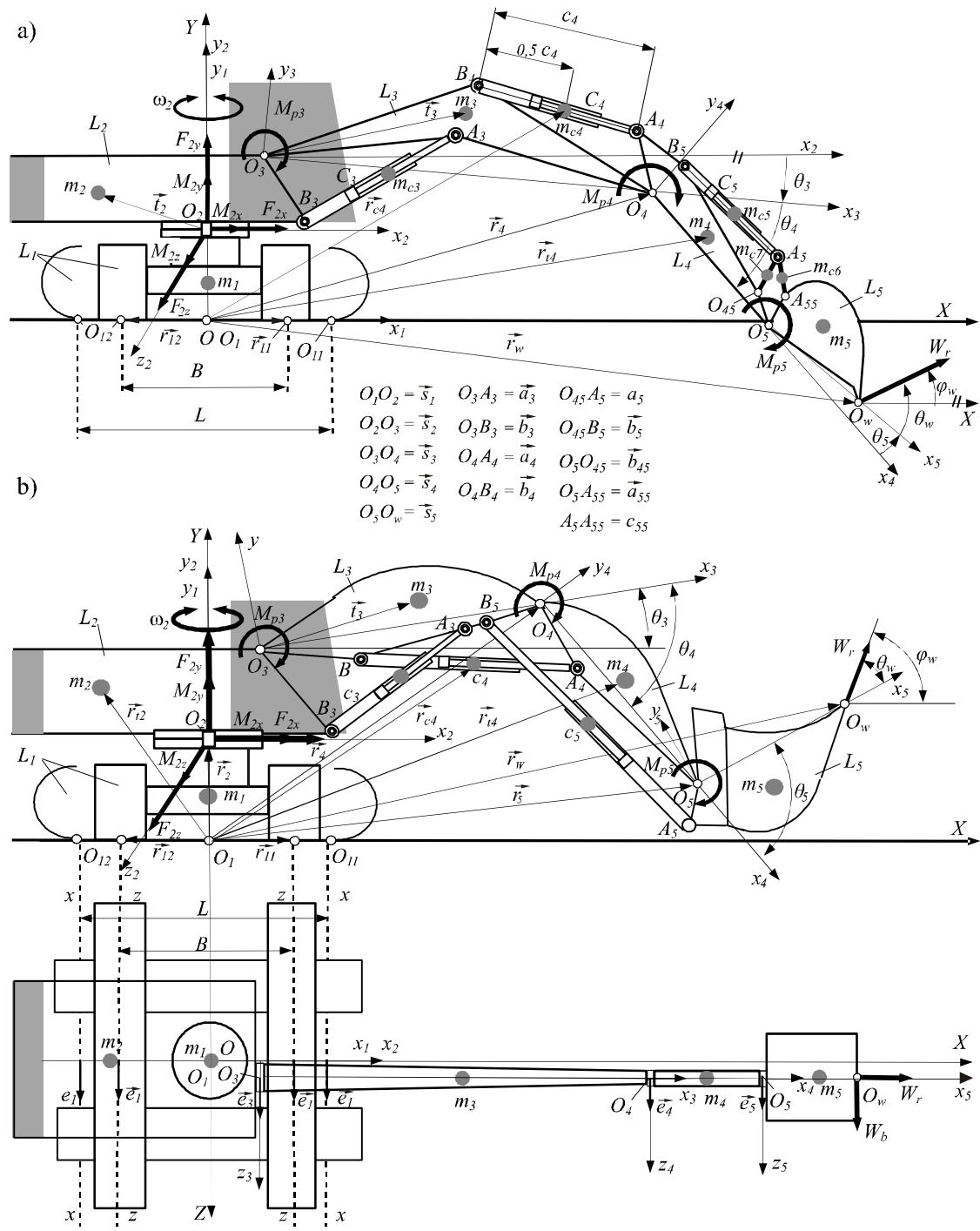

Figure 2

Determining the load of axial bearing slewing platforms of hydraulic excavator with a) backhoe attachment; b) shovel attachment 
The subset of transmission parameters of the drive mechanism of the bucket $C_{5}$ is determined by the set:

- the vectors of the position of joint centres where the hydraulic cylinder is connected to the kinematic chain members, $m_{c i}$ - the mass of the hydraulic cylinder, $n_{c i}$ - the number of hydraulic cylinders of the drive mechanism.

The subset of transmission parameters of the drive mechanism of the bucket $C_{5}$ is determined by the set:

$$
C_{p 5}=\left\{a_{5}, c_{55}, \widehat{\vec{a}}_{55}, \widehat{\vec{b}}_{45}, m_{c 6}, m_{c 7}\right\}
$$

where: $a_{5}$ - the length of the lever of the bucket cylinder in the transmission part of the drive mechanism, Figure. $2 ; c_{55}$ - the length of the link in the transmission part of the drive mechanism; $\widehat{\vec{a}}_{55}, \overrightarrow{\vec{b}}_{45}$ - the coordinates, of the position of the centre of joints in which transmission levers are connected to the links, $m_{c 6}$ - the mass of the link in the transmission part of the drive mechanism, $m_{c 7}$ - the mass of the lever of the bucket cylinder in the transmission part of the drive mechanism.

The assumptions of the mathematical model of the excavator are: 1) the support surface and kinematic chain members are modelled using rigid bodies, 2) the first joint of the kinematic chain between the movement mechanism surface represents a polygon bounded by potential longitudinal $x-x$, Figure 2 and transverse $z-z$ excavator rollover lines. Inside the polygon, the first joint has the shape of a translatory - sliding joint, while on the edges of the polygon it has the shape of rotary joints $O_{11}, O_{12}$, whose axes represent potential excavator rollover lines, 3) during the manipulation task the work of the excavator is stable, i.e. there are no potential movements in the first joint, 4) during the digging operation the kinematic chain of the excavator has an open configuration subjected to: a) gravitational forces (weights) of: kinematic chain members, members of the drive system and material scooped by the bucket and b) digging resistance $W$ in the centre of the bucket cutting edge $O_{w}$, the position of the hydraulic cylinders mass centres is in the middle of the current length of hydraulic cylinders, 5) masses of joint elements belong to the members of the manipulator kinematic chain, 6) the influence of friction is neglected in the kinematic chain joints and drive mechanism joints. The digging resistance vector is determined with the equation:

$$
\vec{W}=W_{r} \cos \varphi_{w} \vec{i}+W_{r} \sin \varphi_{w} \vec{j}+W_{b} \vec{k}
$$

where: $W_{r}$ - the digging resistance which acts in the plane of the manipulator, $W_{b}$ the lateral digging resistance, $\varphi_{w}$ - the angle of the direction in which the digging resistance $W_{r}$ acts in relation to the horizontal $O X Z$ plane of the absolute coordinate system. The direction in which the digging resistance $W_{r}$ acts in relation to the horizontal $O X Z$ plane of the absolute coordinate system is determined with the angle: 
$\varphi_{i}=\sum_{3}^{i} \theta_{i}+\theta_{w} \quad \forall i=3,4,5$

where: $\theta_{i}(i=3,4,5)$ - the angle of the relative position of member $L_{i}$ in relation to the previous member $L_{i-1}$ upon the rotation around the axis of joint $O_{i}$ by changing the length $c_{i}$ of the drive mechanism hydraulic cylinder, $\theta_{w}$ - the angle of the direction in which the digging resistance acts in relation to the positive $O_{5} x_{5}$ axis of the local coordinate system of the bucket $L_{5}$.

The magnitude of the digging resistance $W_{r}$ vector, for a particular direction of action, is defined by the equation [15]:

$$
W_{r}=\min \left\{W_{o}, W_{1}, W_{3}, W_{4}, W_{5}\right\}
$$

where: $W_{o}$ - the highest boundary digging resistance determined from the excavator non-sliding conditions in the plane of the support surface, $W_{l}$ - the highest boundary digging resistance determined from the given excavator stability conditions for potential rollover lines, $W_{3}, W_{4}, W_{5}$ - are the highest boundary values of the digging resistance which can be overcome by the drive mechanisms of manipulator boom, stick, and bucket at the maximum pressure of the excavator hydraulic system.

The unit vector of the digging resistance $W_{r}$ :

$$
\operatorname{ort} \vec{W}_{r}=\cos \varphi_{w} \vec{i}+\sin \varphi_{w} \vec{j}
$$

The boundary digging resistance $W_{o}$ bounded by the force of adherence of the excavator to the support surface is determined from the balance conditions of the sliding part of the first joint, i.e. from the condition that the support and movement mechanism of the excavator will not slide, during digging, along the support surface:

$W_{o}=\frac{m g \cdot \mu_{p}}{\left|\cos \varphi_{w}\right|}$

where: $m$ - the total mass of the excavator, $\mu_{p}$ - the coefficient of adherence of the excavator movement mechanism to the support surface.

Depending on the position of the kinematic chain of the excavator and, the boundary digging resistance $W_{l}$, which is limited by the static stability of the excavator, is determined from the balance conditions for one of the rotary joints $O_{11}, O_{12}$, whose axes represent the potential excavator rollover lines, Figure 2: 


$$
W_{1}=\left\{\begin{array}{c}
W_{11}=\frac{-M_{o 11}}{\left(\left(\vec{r}_{w}-\vec{r}_{11} \times \operatorname{ort} \vec{W}_{r}\right) \cdot \vec{e}_{1}\right)}, \\
\forall y_{w}>0, \varphi_{12}>\varphi_{w}>\left(\varphi_{11}+180^{\circ}\right), \\
\forall y_{w}<0, \varphi_{11}>\varphi_{w}>\left(\varphi_{12}+180^{\circ}\right), \\
\quad-M_{o 12} \\
W_{12}=\frac{\left(\left(\vec{r}_{w}-\vec{r}_{12} \times \operatorname{ort} \vec{W}_{r}\right) \cdot \vec{e}_{1}\right),}{\forall y_{w}>0\left(\varphi_{12}+180^{\circ}\right)>\varphi_{w}>\varphi_{11},} \\
\forall y_{w}<O\left(\varphi_{11}-180^{\circ}\right)>\varphi_{w}>\varphi_{12},
\end{array}\right.
$$

where: $\vec{e}_{1}=\{0,0,1\}$ - the unit vector of the first rotary joint (for the longitudinal $x$ - $x$ or transverse $z-z$ excavator rollover line), $M_{o 11}, M_{o l 2}$ - the gravitational moments for potential excavator rollover lines, i.e. rotary joints $O_{11}, O_{12}, \vec{r}_{w}$ - the vector of the position of the bucket cutting edge centre, $\vec{r}_{11}, \vec{r}_{12}$ - the vectors of the position of the centre of the appropriate first rotary joint $O_{11}, O_{12}, y_{w}$-the vertical coordinate of the bucket top, $\varphi_{11}, \varphi_{12}$ - the angles of the position of vectors and in relation to the horizontal plane $O X Z$, determined by the equations:

$$
\begin{aligned}
& \varphi_{11}=\arccos \left(\frac{\left(\vec{r}_{w}-\vec{r}_{11}\right) \cdot \vec{i}}{\left|\vec{r}_{w}-\vec{r}_{11}\right|}\right), \\
& \varphi_{12}=\arccos \left(\frac{\left(\vec{r}_{w}-\vec{r}_{12}\right) \cdot \vec{i}}{\left|\vec{r}_{w}-\vec{r}_{12}\right|}\right)
\end{aligned}
$$

Gravitational moments for potential excavator rollover lines, i.e. rotary joints $O_{11}, O_{12}$ :

$$
M_{o 1}=\left\{\begin{array}{l}
M_{o 11}=-g \sum_{k=1}^{k=5} m_{k}\left(\left(\vec{r}_{t k}-\vec{r}_{11}\right) \times \vec{j}\right) \cdot \vec{e}_{1}-g \sum_{k=3}^{k=7} m_{c k}\left(\left(\vec{r}_{c t k}-\vec{r}_{11}\right) \times \vec{j}\right) \cdot \vec{e}_{1}, \\
M_{o 12}=-g \sum_{k=1}^{k=5} m_{k}\left(\left(\vec{r}_{t k}-\vec{r}_{12}\right) \times \vec{j}\right) \cdot \vec{e}_{1}-g \sum_{k=3}^{k=7} m_{c k}\left(\left(\vec{r}_{c t k}-\vec{r}_{12}\right) \times \vec{j}\right) \cdot \vec{e}_{1}
\end{array}\right.
$$

where: $m_{k}$ - the mass of the kinematic chain members, $m_{c k}$ - the mass of the drive mechanism members, $\vec{r}_{t k}$ - the vector of the position of the mass centre of kinematic chain members, $\vec{r}_{c t k}$ - the vector of the position of the mass centre of drive mechanism members.

Boundary digging resistances Wi $(i=3,4,5)$ which can be overcome by the drive mechanisms of the manipulator, for the known and the position of the excavator 
kinematic chain upon the action of the maximum drive moments $\mathrm{Mpi}$, are determined from the balance conditions for the manipulator joints Oi axes, Fig. 2:

$W_{i}=\frac{-M_{p i}-M_{r i}}{\left(\left(\vec{r}_{w}-\vec{r}_{i}\right) \times \operatorname{ort} \vec{W}_{r}\right) \cdot \vec{e}_{i}} \forall i=3,4,5$

where: $M_{p i}$ - the maximum drive moments of manipulator mechanisms for both directions in which they act (upon piston pushing and piston pulling in the hydraulic cylinder), $M_{r i}$ - the moment of gravitational forces of the kinematic chain members, members of the excavator drive mechanisms, and the mass of soil scooped by the full bucket, for certain axes of joints $O_{i}, \vec{r}_{i}$ - the vector of the position of the joint centre in the excavator kinematic chain, $\vec{e}_{i}=\{0,0,1\}$ - the unit vector of the joint axes in the manipulator kinematic chain.

The maximum drive moments of manipulator mechanisms for both directions in which they act (upon piston pushing and piston pulling in the hydraulic cylinder):

$$
M_{p i}=\left\{\begin{array}{l}
M_{p i 1}=\operatorname{sign}\left(\dot{\theta}_{i}\right) \cdot r_{c i} \cdot n_{c i} \cdot\left[\frac{d_{i 1}^{2} \pi}{4} p_{m}-\frac{\left(d_{i 1}^{2}-d_{i 2}^{2}\right) \pi}{4} p_{o}\right] \cdot \eta_{c i} \\
\forall i=3,4,5 ; \dot{\theta}_{3}>0, \dot{\theta}_{4}<0, \dot{\theta}_{5}>0 \\
M_{p i 2}=\operatorname{sign}\left(\dot{\theta}_{i}\right) \cdot r_{c i} \cdot n_{c i} \cdot\left[\frac{\left(d_{i 1}^{2}-d_{i 2}^{2}\right) \pi}{4} p_{m}-\frac{d_{i 1}^{2} \pi}{4} p_{o}\right] \cdot \eta_{c i} \\
\forall i=3,4,5 ; \dot{\theta}_{3}<0, \dot{\theta}_{4}>0, \dot{\theta}_{5}<0,
\end{array}\right.
$$

where: $\dot{\theta}_{i}$ - the angular velocities of the kinematic chain members, $r_{c i}$ - the transmission function of the drive mechanism which depends on the length of the hydraulic cylinder and the vector, i.e. coordinates, of the position of the joint centres where hydraulic cylinders are connected to the members of the drive mechanism kinematic chain, $p_{m}$ - the maximum duct pressure during the extension stroke of the hydraulic cylinder, $p_{o}$ - the maximum duct pressure during the retraction stroke of the hydraulic cylinder, $\eta_{c i}$ - the mechanical degree of the hydraulic cylinder efficiency.

The moment of the gravitational forces of the kinematic chain members, members of the excavator drive mechanisms, and the mass of soil scooped by the full bucket, for certain axes of joints $O_{i}$, is determined by the equation:

$$
M_{r i}=M_{o i}-g m_{z}\left(\left(\vec{r}_{t 5}-\vec{r}_{i}\right) \times \vec{j}\right) \cdot \vec{e}_{i} \quad \forall i=1,3,4,5
$$

where: $M_{o i}$ - the moment of the gravitational forces of the kinematic chain members and members of the excavator drive mechanisms for certain axes of joints $O_{i}, m_{z}$ - the mass of the material scooped with the bucket, where it is assumed that the centre of the scooped material mass overlaps with the centre of the bucket mass. 
The moment of the gravitational forces of the kinematic chain members and members of the excavator drive mechanisms for certain axes of joints $O_{i}$, when the bucket is empty, is determined by the equation, Figure 2 :

$$
M_{o i}=-g \sum_{k=i}^{k=5} m_{k}\left(\left(\vec{r}_{t k}-\vec{r}_{i}\right) \times \vec{j}\right) \cdot \vec{e}_{i}+M_{o c i} \forall i=3,4,5
$$

where: $M_{o c i}$ - the moment of the gravitational forces of the excavator drive mechanism members for certain axes of joints $O_{i}(i=3,4,5)$.

The moments of the gravitational forces of the excavator drive mechanism members for certain axes of joints $O_{i}(i=3,4,5)$ are determined by the following equations:

$$
M_{o c i}= \begin{cases}M_{o c 3}=-g \frac{n_{c 3} m_{c 3}}{2}\left(\left(\vec{r}_{A 3}-\vec{r}_{3}\right) \times \vec{j}\right) \cdot \vec{e}_{3}- \\ -g \sum_{k=4}^{k=7} n_{c k} m_{c k}\left(\left(\vec{r}_{c t k}-\vec{r}_{3}\right) \times \vec{j}\right) \cdot \vec{e}_{3} & \forall i=3 \\ M_{o c 4}=-g \frac{n_{c 4} m_{c 4}}{2}\left(\left(\vec{r}_{A 4}-\vec{r}_{4}\right) \times \vec{j}\right) \cdot \vec{e}_{4}- \\ -g \sum_{k=5}^{k=7} n_{c k} m_{c k}\left(\left(\vec{r}_{c t k}-\vec{r}_{4}\right) \times \vec{j}\right) \cdot \vec{e}_{4} & \forall i=4 \\ M_{o c 5}=-g \frac{m_{c 6}}{2}\left(\left(\vec{r}_{A 5}-\vec{r}_{5}\right) \times \vec{j}\right) \cdot \vec{e}_{5} & \forall i=5\end{cases}
$$

where: $\vec{r}_{A 3}, \vec{r}_{A 4}, \vec{r}_{A 5}$ - the coordinates of joints where hydraulic cylinders are connected to the kinematic chain members, Figure 2.

Depending on the position of the bucket, the mass of the material scooped by the bucket is defined by the expression:

$$
m_{z}= \begin{cases}\rho_{z} \cdot V \cdot\left|\cos \varphi_{5}\right| & \forall 270^{\circ} \geq \varphi_{5} \geq 90^{\circ} \\ 0 & \forall 270^{\circ}<\varphi_{5}<90^{\circ}\end{cases}
$$

where: $\rho_{z}$ - the density of the material, $V$ - the volume of the bucket. The value of the lateral digging resistance $W_{b}$, for a particular position of the excavator kinematic chain, is defined by the equation:

$W_{b}=\frac{m \cdot g \cdot L}{4 \cdot x_{w}} \mu_{o}$

where: $m$ - the mass of the excavator, $L$ - the length of the continuous tracks footprint in Figure 2, $\mu_{o}$ - the coefficient of the turning resistance of the tracks against the excavator support surface, $x_{w}$ - the horizontal coordinate of the bucket cutting edge centre. 


\section{Bearing Loads}

The fictive interruption of the kinematic chain of the excavator in the joint $O_{2}$ of the slewing platform $L_{2}$ and the reduction of all loads, of the removed part, into its centre, yield:

- the resulting force which subjects the axial bearing to loading:

$\vec{F}_{2}=\vec{W}-g \sum_{i=2}^{5} m_{i} \vec{j}-g \sum_{i=3}^{7} m_{c i} \vec{j}-g m_{z} \vec{j}$

- $\quad$ and the resulting moment which subjects the axial bearing to loading:

$$
\begin{aligned}
& \vec{M}_{2}=\left(\left(\vec{r}_{w}-\vec{r}_{2}\right) \times \vec{W}\right)-g \sum_{i=2}^{5} m_{i}\left(\left(\vec{r}_{t i}-\vec{r}_{2}\right) \times \vec{j}\right)- \\
& -g m_{z}\left(\left(\vec{r}_{t 5}-\vec{r}_{2}\right) \times \vec{j}\right)-g \sum_{i=3}^{i=7} n_{c i} m_{c i}\left(\left(\vec{r}_{c t i}-\vec{r}_{2}\right) \times \vec{j}\right)
\end{aligned}
$$

where: $\vec{r}_{2}$ - the vector of the position of the joint centre (axial bearing) $\mathrm{O}_{2}$.

Components of force $F_{2}$ of joint $\mathrm{O}_{2}$ along the coordinate axes:

$$
F_{2 x}=\vec{F}_{2} \cdot \vec{i}, \quad F_{2 y}=\bar{F}_{2} \cdot \vec{j}, \quad F_{2 z}=\vec{F}_{2} \cdot \vec{k}
$$

Components of moment $M_{2}$ of joint $O_{2}$ along the coordinate axes:

$$
M_{2 x}=\vec{M}_{2} \cdot \bar{i}, \quad M_{2 y}=\vec{M}_{2} \cdot \vec{j} \quad M_{2 z}=\vec{M}_{2} \cdot \vec{k}
$$

Components of axial bearing loads of the excavator slewing platform are:

- axial force:

$$
F_{2 a}=F_{2 y}
$$

- radial force:

$F_{2 r}=\left(F_{2 x}^{2}+F_{2 z}^{2}\right)^{0,5}$

- $\quad$ and moment:

$$
M_{2 r}=\left(M_{2 x}^{2}+M_{2 z}^{2}\right)^{p, 5}
$$

Moment $M_{2 r}$, whose vector lies in the horizontal plane, subjects the axial bearing to loading, while moment $M_{2 y}$, whose vector direction matches the bearing axis, balances the drive moment of the platform rotation mechanism. The size of the bearing is selected on the basis of the determined equivalent spectrum of bearing loads and diagrams of bearing loading capacity (curves I, II, III, IV and V, Fig. 4), which are provided by the specialized bearing manufacturers [16]. 
The equivalent spectrum of bearing loads consists of an equivalent force and an equivalent bearing load moment determined by the equations for:

- $\quad$ equivalent force $F_{e}$ :

$F_{e}=\left(a \cdot F_{2 a}+b \cdot F_{2 r}\right) f_{s}$

- $\quad$ and equivalent moment $M_{e}$ :

$M_{e}=f_{s} \cdot M_{2 r}$

where: $a$ - the factor of the axial force influence, $b$ - the factor of the radial force influence, $f_{s}$ - the factor of the bearing working conditions. Values of factors $a, b, f_{s}$ are provided by the bearing manufacturers depending on the type of bearing (single-row, multi-row, ball, roller), type and size of machines and their working conditions.

\section{Selection of Bearings}

For a reliable selection of an axial bearing of a slewing platform in a hydraulic excavator, of a certain size, it is necessary to determined the spectra of bearing loads for all possible configurations of kinematic chains that the excavator is equipped with. These possible configurations of kinematic chains differ from the variants of support and movement mechanisms, then the variants of manipulator members, and the tools which the excavator uses. Furthermore, it is also necessary to determine the spectrum of bearing loads for the same configuration of the excavator kinematic chain in as many positions of the entire working range of the excavator as possible, having in mind that each position of the kinematic chain carries the possibility of the action of the digging resistance in various directions depending on the excavator working conditions. To satisfy all of the above requirements, on the basis of the previously given calculation procedure, a computer programme was developed to determine the loading spectrum and select the axial bearing of the platform rotation drive in hydraulic excavators.

During the analysis the following is set at the programme input: $L_{i}$ - parameters of the members of the excavator kinematic chains, $C_{i}$ - parameters of the drive mechanisms of the excavator manipulator, $p_{m}$ - the maximum pressure of the hydraulic static system of the excavator, $p_{o}$ - the pressure in the retraction duct of the hydraulic static system of the excavator, $N_{3}$ - the desired number of the manipulator boom positions in its range of movement, $N_{4}$ - the desired number of the stick positions in its range of movement for a certain position of the manipulator boom, $N_{5}$ - the desired number of the bucket positions in its range of movement for a certain position of the manipulator stick, $N_{w}$ - the desired number of changes in the angle $\theta_{w}$ of the directions in which the digging resistance acts for a certain position of the bucket, $\theta_{w p}$ - the initial angle of the direction in which the 
digging resistance acts, $\theta_{w k}$ - the final angle of the direction in which the digging resistance acts, $\rho_{z}$ - the density of the scooped material, $V_{z}$ - the volume of the bucket, $\mu_{p}$ - the coefficient of adherence, $a$ - the factor of the influence of the axial bearing force, $b$ - the factor of the influence of the radial bearing force, $f_{s}$ - the factor of the bearing working conditions.

Based on the input values, and through the cyclic change of the given numbers $N_{w}, N_{5}, N_{4}$ and $N_{3}$, Figure 3, the programme determines: a) geometric values $\left(\theta_{i}\right.$, $r_{i}, r_{t i}, r_{w}$ ) which define the position of the joint centres and mass centres of the excavator kinematic chain, b) loading moments $\left(M_{o i}, M_{r i}\right)$ and drive moment $\left(M_{p i}\right)$ of drive mechanisms, c) boundary digging resistances $\left(W_{o}, W_{1}, W_{3}, W_{4}, W_{5}\right)$, for the entire working range of the excavator, d) components of axial bearing loads $\left(F_{2 a}\right.$, $\left.F_{2 r}, M_{2 r}\right)$, and e) equivalent axial bearing loads $\left(F_{e}, M_{e}\right)$.

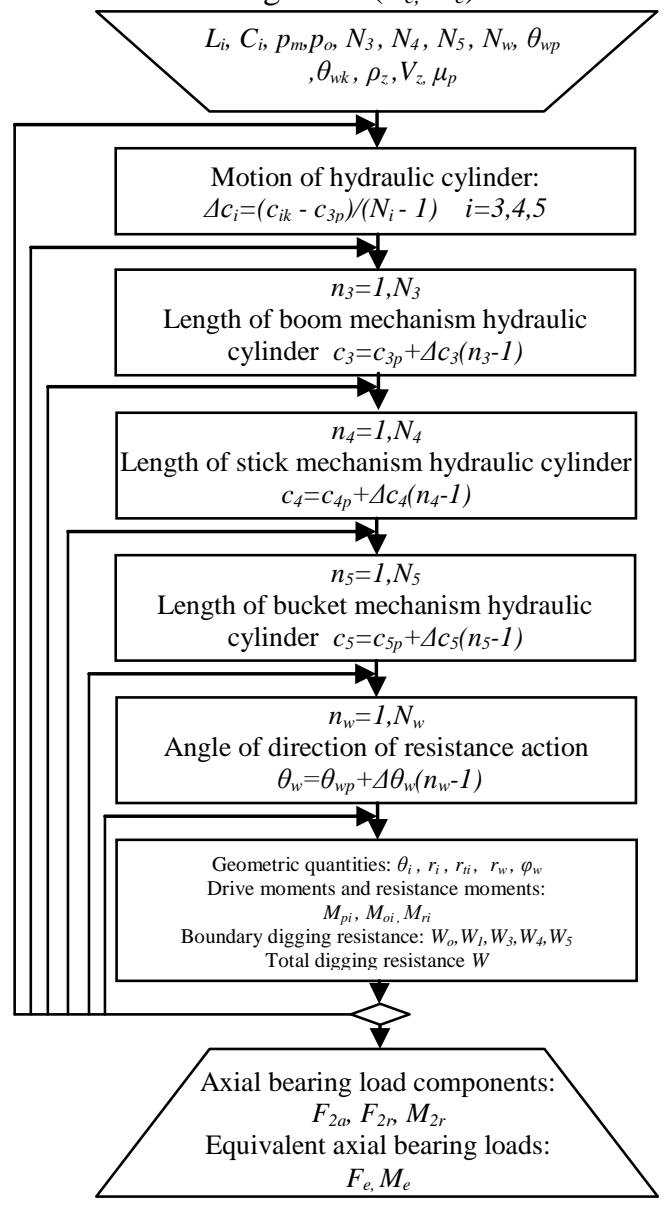

Figure 3

Algorithm of the programme for the analysis of axial bearing loads of a slewing platform drive in hydraulic excavators 
The programme output yields a spectrum of bearing loads for the entire working range of the excavator which consists of equivalent bearing loads for each position of the excavator kinematic chain and for each given direction in which the digging resistance acts. By comparing the spectrum of the equivalent bearing loads obtained through analysis with the permitted bearing loads provided in the bearing loading capacity diagrams, the reliably necessary size of the bearing is selected.

\section{Results and Discussion}

By using the developed software, the analysis was conducted for the axial bearing load of a slewing platform drive in a hydraulic excavator with the mass of 100000 $\mathrm{kg}$ and the power of $400 \mathrm{~kW}$ with a backhoe and a shovel attachment.

The kinematic chain of the selected size of the excavator with a backhoe attachment can be equipped with: three different support and movement mechanisms $L_{1}$, Figure 2, three booms $L_{3}$ and four sticks $L_{4}$ of various lengths, and twelve backhoe attachments $L_{5}$ of various volumes for the digging of materials of different characteristics in different working conditions

The kinematic chain of the selected size of the excvator with a shovel attachment can be equipped with two different support and movement mechanisms $L_{l}$ and six shovel attachments $L_{5}$ of various volumes. As an example, the spectrum of axial bearing load of a slewing platform drive was determined for two possible variants $\mathrm{A} 1$ and $\mathrm{A} 2$ of the kinematic chain of the excavator with a backhoe attachment and for two possible variants B1 and B2 of the kinematic chain of the excavator with a shovel attachment.

Variant A1 consists of a support and movement mechanism with the footprint length of $\mathrm{L}=4.64 \mathrm{~m}$, Figure 2, track distance of $\mathrm{B}=3.6 \mathrm{~m}$, boom with the length of $s_{3}=7.2 \mathrm{~m}$, stick with the length of $\mathrm{s}_{4}=2.9 \mathrm{~m}$, and a backhoe attachment with the volume of $V=4.8 \mathrm{~m}^{3}$ for the digging of a material with the density of $\rho_{z}=2200$ $\mathrm{kg} / \mathrm{m}^{3}$.

Variant A2 consists of a support and movement mechanism with the footprint length of $\mathrm{L}=5.035 \mathrm{~m}$, track distance of $\mathrm{B}=3.6 \mathrm{~m}$, boom with the length of $\mathrm{s}_{3}=10.5$ $\mathrm{m}$, stick with the length of $\mathrm{s}_{4}=5.8 \mathrm{~m}$, and a backhoe attachment with the volume of $\mathrm{V}=2.0 \mathrm{~m}^{3}$ for the digging of a material with the density of $\rho_{\mathrm{z}}=1800 \mathrm{~kg} / \mathrm{m}^{3}$.

Variant B1 consists of a support and movement mechanism with the footprint length of $\mathrm{L}=4.64 \mathrm{~m}$, track distance of $\mathrm{B}=3.6 \mathrm{~m}$, boom with the length of $\mathrm{s}_{3}=4.65$ $\mathrm{m}$, stick with the length of $\mathrm{s}_{4}=3.4 \mathrm{~m}$, and a shovel attachment with the volume of $\mathrm{V}=4.4 \mathrm{~m}^{3}$ for the digging of a material with the density of $\rho_{\mathrm{z}}=2200 \mathrm{~kg} / \mathrm{m}^{3}$.

Variant B2 consists of a support and movement mechanism with the footprint length of $\mathrm{L}=5.035 \mathrm{~m}$, track distance of $\mathrm{B}=3.6 \mathrm{~m}$, boom with the length of $\mathrm{s}_{3}=4.65$ 
$\mathrm{m}$, stick with the length of $\mathrm{s}_{4}=3.4 \mathrm{~m}$, and a shovel attachment with the volume of $\mathrm{V}=6.5 \mathrm{~m}^{3}$ for the digging of a material with the density of $\rho_{\mathrm{z}}=1650 \mathrm{~kg} / \mathrm{m}^{3}$.

The input file of the programme contains the parameters shown in Table 1 which are the same for all variants A1, A2, B1, and B2 of the kinematic chain of the excavator.

Table 1

Values of the input parameters of the programme

\begin{tabular}{|l|c|c|c|c|c|c|c|c|c|c|}
\hline Parameters & $\mathrm{p}_{\mathrm{m}}$ & $\mathrm{p}_{\mathrm{o}}$ & $\mathrm{N}_{3}$ & $\mathrm{~N}_{4}$ & $\mathrm{~N}_{5}$ & $\mathrm{~N}_{\mathrm{w}}$ & $\mu_{\mathrm{p}}$ & $\mathrm{a}$ & $\mathrm{b}$ & $\mathrm{f}_{\mathrm{s}}$ \\
\hline Values & $\begin{array}{c}32 \\
\mathrm{MPa}\end{array}$ & $\begin{array}{c}1.2 \\
\mathrm{MPa}\end{array}$ & 30 & 20 & 10 & 10 & 0.85 & 1 & 2.05 & 1.45 \\
\hline
\end{tabular}

The determination of the spectrum of bearing loads of variants A1 and A2 includes the range of the change in the acting angle of a possible digging resistance between $\theta_{w p}=30^{\circ}$ and $\theta_{w k}=150^{\circ}$. The determination of the spectrum of bearing loads of variants B1 and B2 includes the range of the change in the acting angle of a possible digging resistance between $\theta_{w p}=200^{\circ}$ and $\theta_{w k}=300^{\circ}$.

On the basis of the set input parameters, using the developed programme, the spectrum of axial bearing loads was determined for the slewing platform drive for variants A1,A2, B1 and B2 of the excavator kinematic chain. The programme output provided, among other things, the equivalent force and the equivalent bearing load moment for variants $\mathrm{A} 1, \mathrm{~A} 2, \mathrm{~B} 1$ and $\mathrm{B} 2$ of the excavator kinematic chain, determined for $6000\left(\mathrm{~N}_{3} \mathrm{xN}_{4} \mathrm{XN}_{5}\right)$ positions in the entire working range and in each position for 10 different directions in which potential digging resistances could act.

The obtained values of the equivalent forces and moments are shown in the form of a diagram shown in Figure 4, Figure 5 as a spectrum of bearing loads for the longitudinal ( $\mathrm{x}-\mathrm{x})$ and transverse ( $\mathrm{z}-\mathrm{z})$ potential rollover lines for variants $\mathrm{A} 1, \mathrm{~A} 2$, $\mathrm{B} 1$ and $\mathrm{B} 2$ of the excavator kinematic chain. All of the diagrams of the spectra of axial bearing loads for variants A1, A2, B1 and B2 of the excavator kinematic chain show diagrams of the permitted loading capacity of the five same bearing which differ in size are shown in Figure 4, curves I, II, III, IV, V) [16]. The diagram of the permitted loading capacity represents the dependency of the allowed axial bearing moment and force. The slewing platform drive corresponds to that size of the bearing whose permitted loading capacity is closest yet larger than the potential values of the spectrum of the equivalent bearing loads.

For example, by comparing the spectra of the equivalent bearing loads for variant A1 of the excavator kinematic chain with the loading capacity diagrams it can be noticed that the potential longitudinal rollover line $(\mathrm{x}-\mathrm{x})$, Figure 4a corresponds to the bearing size V, while for variant A2 the transverse rollover line (z-z), Figure $4 \mathrm{~b}$ corresponds to the bearing size II. 
As far as variant B1 of the excavator kinematic chain is concerned, the potential longitudinal rollover line $(\mathrm{x}-\mathrm{x})$, Figure $5 \mathrm{c}$, corresponds to the bearing size IV, while for variant B2 the transverse rollover line (z-z), Figure 5d, corresponds to the bearing size II.

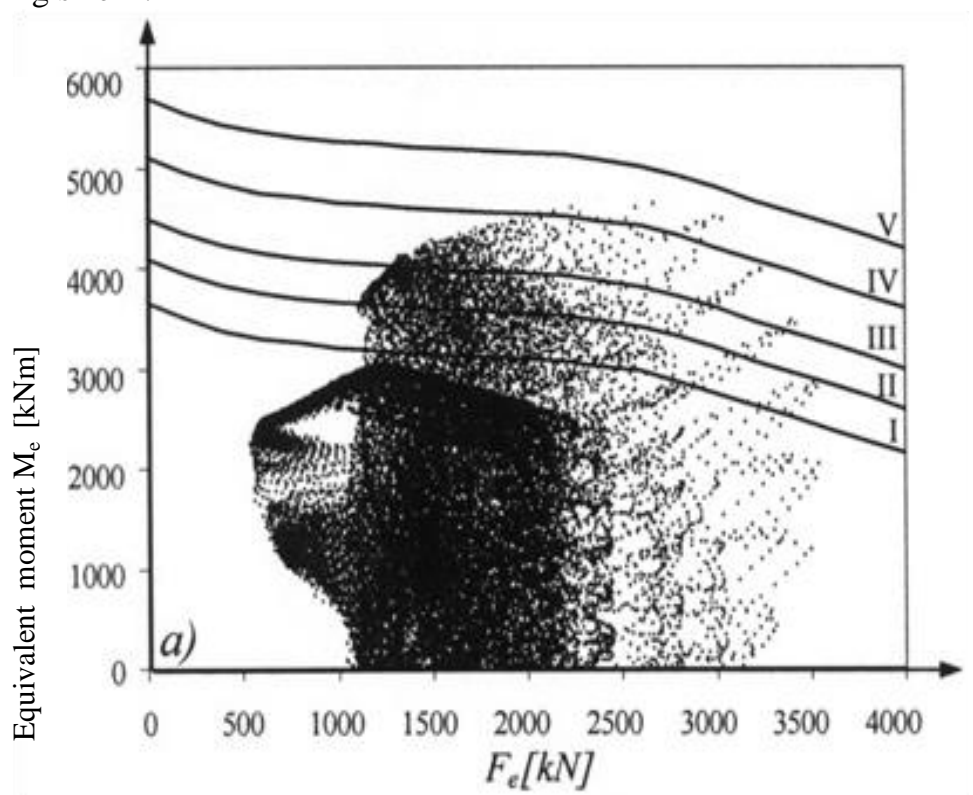

Equivalent force $\mathrm{F}_{\mathrm{e}}[\mathrm{Nm}]$

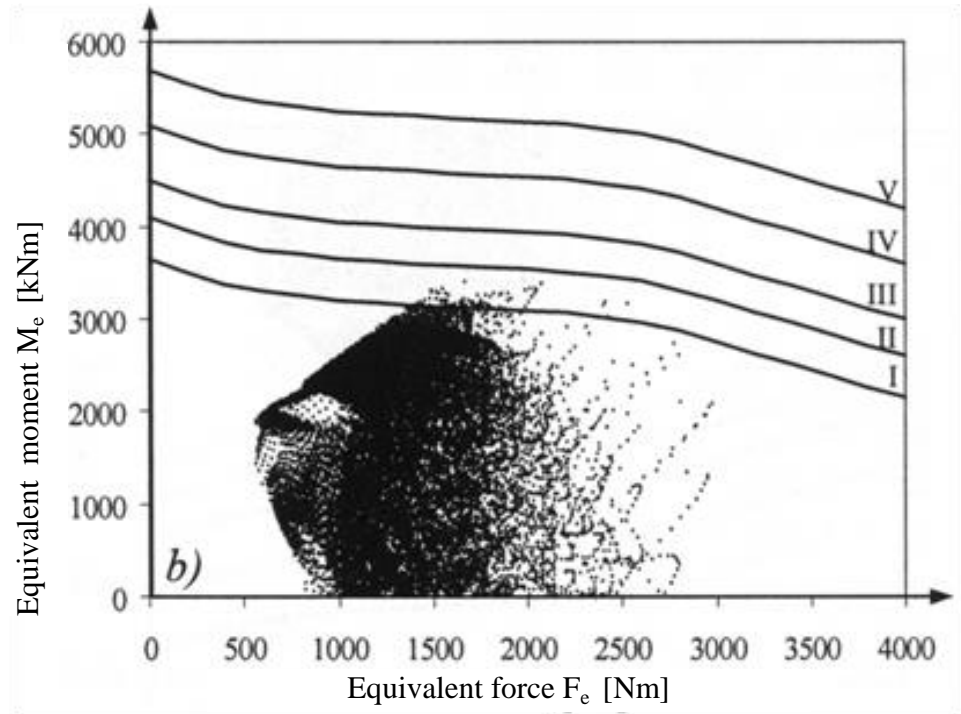

Figure 4

Spectrums of axial bearing load of a slewing platform drive of hydraulic excavators: a) variant A1 longitudinal rollover line $\mathrm{x}-\mathrm{x}, \mathrm{b}$ ) variant $\mathrm{A} 2$ - transverse rollover line $\mathrm{z}-\mathrm{z}$ 

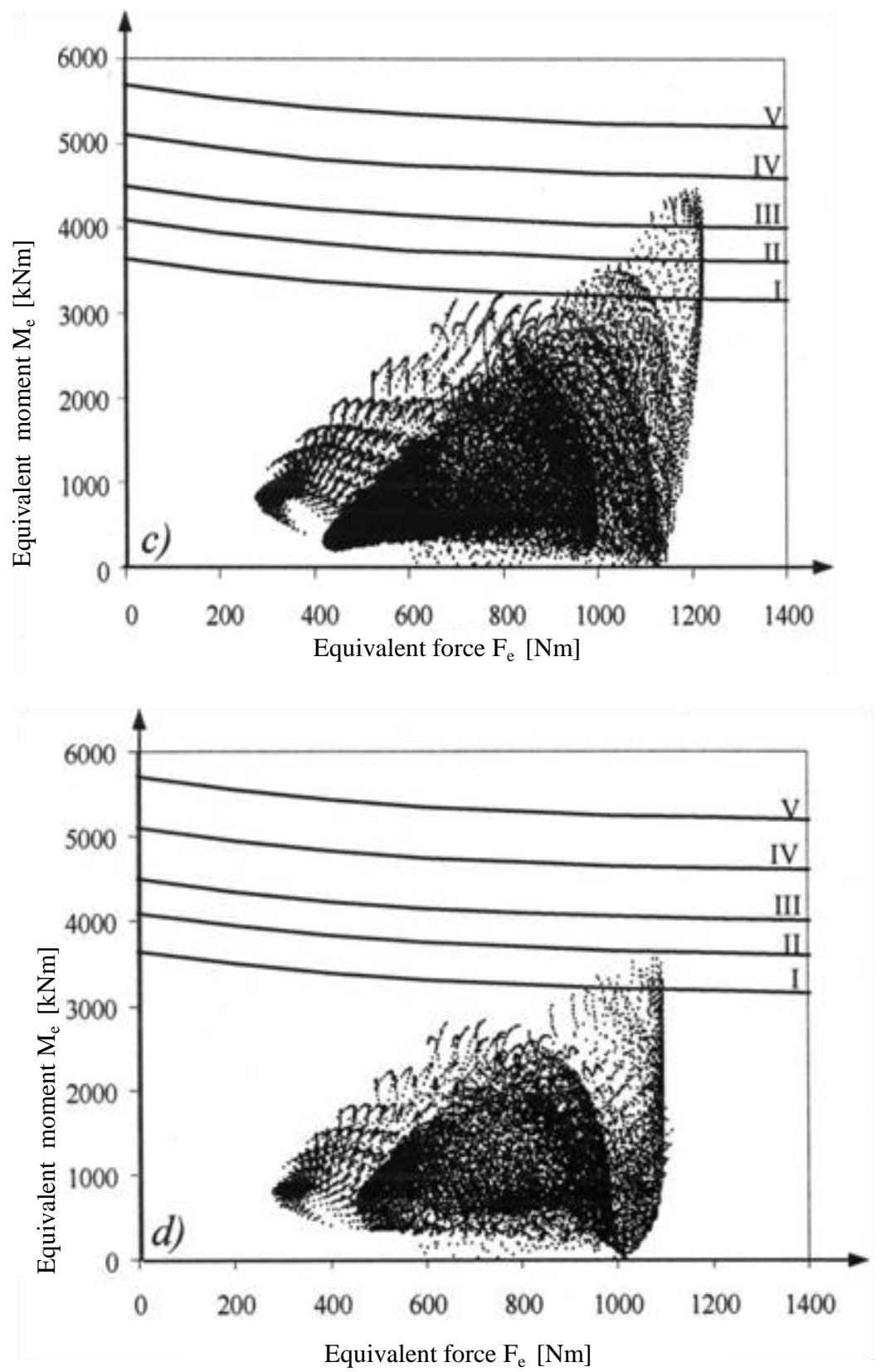

Figure 5

Spectrums of axial bearing load of a slewing platform drive of hydraulic excavators: c) variant B1 longitudinal rollover line $\mathrm{x}-\mathrm{x}, \mathrm{d}$ ) variant $\mathrm{B} 2$ - transverse rollover line $\mathrm{z}-\mathrm{z}$ 
Finally, on the basis of the obtained loading spectra and diagrams of permitted bearing loading (Figs. 4, 5), the analyzed excavator variants A1, A2 and B1, B2 correspond to the bearing size $\mathrm{V}$ which belongs to the KD 800 series of single-row roller slewing bearings with internal toothing manufactured by Rothe Erde [16].

\section{Conclusions}

The synthesis procedure for the slewing platform drive of hydraulic excavators should, among other things, provide for the selection of the axial bearing size. It is characteristic for all sizes of hydraulic excavators that the same model of excavator can have different configurations of kinematic chains equipped with various working tools. Furthermore, an excavator with the same configuration of the kinematic chain has a number of different positions and working conditions during its operation in its working range. For these reasons, this paper defines a mathematical model upon which software was developed that enables a comprehensive analysis of equivalent axial bearing loads of an excavator slewing platform which allow for a reliable selection of the bearing size. The comprehensive analysis using the developed software includes the determination of the spectra of equivalent axial bearing loads for a slewing platform in each possible configuration of the excavator kinematic chain for a desired number of working positions in the entire working range and in every position for a desired number of different working manners and conditions.

\section{Acknowledgement}

The paper was done within the project TR 35049 financed by the Ministry of Education and Science of the Republic of Serbia.

\section{References}

[1] Maciejewski, J., Jarzebowski, A., Trampczynski, W. (2004) Study on the Efficiency of the Digging Process using the Model of Excavator Bucket, Journal of Terramechanics, Vol. 40, pp. 221-233

[2] Tanasijevic, M., Ivezic, D., Ignjatovic, D. and Polovina, D. (2011) Dependability as Criteria for Bucket Wheel Excavator Revitalization, Journal of Scientific \& Industrial Research (JSIR), Vol. 70, pp. 13-19

[3] Gu, J., Taylor, J., Seward, D. (2007) Modelling of an Hydraulic Excavator using Simplified Refined Instrumental Variable (SRIV) Algorithm, Journal of Control Theory and Applications, No. 5-4, pp. 391-396

[4] Hall, A. S., McAree, P. R. (2005) Robust Bucket Position Tracking for a Large Hydraulic Excavator, Mechanism and Machine Theory, Vol. 140, pp. $1-16$

[5] Plonecki, L., Trampczynski, W., Cendrowicz, J. (1998) A Concept of Digital Control System to Assist the Operator of Hydraulic Excavators, Automation in Construction, Vol. 7, pp. 401-411 
[6] Geu, F., Kecskemethy, F., Pottker, A. (2007) Workspace Analysis and Maximal Force Calculation of a Face-Shovel Excavator using Kinematical Transformers, $12^{\text {th }}$ IFToMM World Congress, Besancon, pp. 18-21

[7] Sung-Uk, L., Chang, P. H. (2002) Control of a Heavy-Duty Robotic Excavator using Time Delay Control with Integral Sliding Surface, Control Engineering Practice 10 697-711

[8] Tadeusz, S., Damian, D., Mariusz, S., (2008) Evaluation of Load Distribution in the Superstructure Rotation Joint of Single-Bucket Caterpillar Excavators, Automation in Construction, Vol. 17-3, pp. 218-223

[9] Jose, I. A., Xabie, S., Jorge, D. (2003) Load Distribution in a Four ContactPoint Slewing Bearing, Mechanism and Machine Theory, Vol. 38-6, pp. 479-496

[10] Hedrih, K., Veljović, Lj. (2013) New Vector Description of Kinetic Pressures on Shaft Bearings of a Rigid Body Nonlinear Dynamics with Coupled Rotations Around No Intersecting Axes, Acta Polytechnica Hungarica, Vol. 10-7, pp. 151-170

[11] Janosevic, D., Nikolic, V., Petrovic, N. (2012) Determining the Load Spectrum of Axial Bearing Slewing Platforms of Hydraulic Excavator, $X X$ International Conference on "Material Handling, Constructions and Logistics", Belgrade, pp. 177-180

[12] Bin, Y., Jiao, Z., Douglas, K., John, L. (1998) High Performance Swing Velocity Tracking Control of Hydraulic Excavators, American Control Conference, Philadelphia, pp. 818-822

[13] Jianqi, L. (1992) An Energy-Saving Device Applied to the Swing System of Hydraulic Excavator, Presented at the International Fluid Power Exposition and Technical Conference, Milwaukee, p. I92-3.2

[14] Jacek, K. (2005) Swing-Free Stop Control of the Slewing Motion of a Mobile Crane, Control Engineering Practice, Vol. 13-4, pp. 451-460

[15] Janosevic, D. (1997) Optimal Synthesis of Drive mechanisms in Hydraulic Excavators. PhD Thesis, Faculty of Mechanical Engineering, University of Nis, Serbia

[16] Slewing Bearings, Rothe Erde GmbH,D-44137 Dortmund (2007) catalog. Available on http://www.thyssenkrupp-rotheerde.com/gb/ produkte_gwl.shtm 